\title{
Effect of oxygen, inoculum composition and flow rate on development of mixed-culture oral biofilms
}

\author{
David J. Bradshaw, ${ }^{1}$ Philip D. Marsh, ${ }^{1}$ Clive Allison ${ }^{2}$ \\ and Kurt M. Schilling ${ }^{2} \dagger$
}

Author for correspondence: David J. Bradshaw. Tel: +44 1980612 732. Fax: +44 1980612731

1 Research Division, CAMR, Salisbury SP4 OJG, UK

2 Unilever Research Port Sunlight Laboratory, Bebington, Wirral

L63 3JW, UK

\begin{abstract}
The effect of aeration on the development of a defined biofilm consortium of oral bacteria was investigated in a two-stage chemostat system. An inoculum comprising 10 species, including both facultatively anaerobic and obligately anaerobic bacteria, and species associated with oral health and disease, was inoculated into an anaerobic first-stage chemostat vessel. The effluent from this chemostat was linked to an aerated [200 ml CO$/$ air $\left.(5: 95, \mathrm{v} / \mathrm{v}) \mathrm{min}^{-1}\right]$ second-stage vessel, in which removable hydroxyapatite discs were inserted to allow biofilm formation. Comparisons were made of planktonic and biofilm communities in the aerated second-stage vessel by means of viable counts. Both planktonic and early biofilm communities were dominated by Neisseria subflava, comprising $>40 \%$ of total c.f.u. in the fluid phase, and $>80 \%$ of c.f.u. in $2 \mathrm{~h}$ biofilms. Obligate anaerobes persisted in this mixed culture, and succession in biofilms led them to predominate only after $7 \mathrm{~d}$. Despite the continuous addition of air, the dissolved oxygen tension $\left(\mathrm{dO}_{2}\right)$ within the culture remained low ( $<5 \%$ of air saturation), and the redox potential $\left(E_{h}\right)$ was $-\mathbf{2 7 5} \mathrm{mV}$. In order to assess the significance of the presence of $N$. subflava in community development, a subsequent experiment omitted this aerobe from the inoculum, to produce a nine-species culture. The planktonic phase was predominated by three streptococcal species, Prevotella nigrescens and Fusobacterium nucleatum. Biofilms again underwent successional changes, with anaerobes increasing in proportion with time. In contrast to the culture including $\mathrm{N}$. subflava, $\mathrm{dO}_{2}$ was $50-60 \%$ of air saturation, and the $E_{h}$ was $+50 \mathrm{mV}$. In the final experiment, the rate of addition of first-stage culture was reduced to 1/10 of that in the previous experiment, in order to determine whether anaerobes were growing, rather than merely persisting in the aerated culture. The data for the planktonic phase indicated that the anaerobes were growing in aerated $\left(\mathrm{dO}_{2} 40-50 \%, E_{\mathrm{h}}+100 \mathrm{mV}\right)$ conditions. Once again, anaerobes increased in proportion in older biofilms. The study indicates that mixed cultures can protect obligate anaerobes from the toxic effects of oxygen, both in the biofilm and planktonic modes of growth.
\end{abstract}

Keywords: biofilm, oxygen, dental plaque microbiology, mixed cultures, chemostat

\section{INTRODUCTION}

Dental plaque is the aetiological agent of two of the most prevalent diseases affecting industrialized societies,

\footnotetext{
† Present address: Unilever Research US Inc., 45 River Road, Edgewater, NJ 07020, USA.

Abbreviation: HA, hydroxyapatite.
}

namely dental caries and periodontal disease. These diseases appear to result from a perturbation of the balance which normally exists between the host and the resident oral microflora. The factors responsible for this perturbation may be external (e.g. diet, antibiotic treatment) or host-related (e.g. immune deficiency, low saliva flow rate). In order to study some of these factors, we have developed model systems incorporating mixed cultures of oral bacteria grown in chemostats, generated 
from inocula of either undefined (Marsh et al., 1983) or defined (McKee et al., 1985; Bradshaw et al., 1989b) composition. The chemostat allows close control of environmental factors and enables single parameters to be varied independently. Thus cause-and-effect relationships may be established between changes in key environmental factors and the composition and metabolism of this model microbial community. We have used this system to study the effects of $\mathrm{pH}$ and carbohydrate pulses (McDermid $e t$ al., 1986; Bradshaw et al., 1989a), fluoride (Bradshaw et al., 1990), antimicrobial agents (McDermid et al., 1987; Bradshaw et al., 1993) and sugar alcohols (Bradshaw \& Marsh, 1994). However, these studies were carried out in a homogeneous chemostat system, while dental plaque is a biofilm. Bacteria in biofilms can have markedly different properties to their planktonic counterparts (Costerton et al., 1987). More recently, therefore, we have modified the chemostat system to allow the development of mixed culture biofilms on hydroxyapatite (HA) discs, which may be removed and replaced aseptically (Bradshaw et al., 1996).

The biofilms that developed in the chemostat system were reproducible both within a chemostat run and between independent chemostat runs. The species composition of biofilms reflected the liquid phase of the culture, and was dominated by anaerobes, even after a few hours (Bradshaw et al., 1996). Such rapid colonization of HA surfaces by anaerobes does not reflect the development of dental plaque observed in vivo (Ritz, 1967; Hardie \& Bowden, 1976), and did not allow the study of succession events during early biofilm formation. In order to attempt to prolong this succession period, a novel two-stage model system was developed, in which the first-stage was a conventional anaerobic chemostat [gas phase: $\mathrm{CO}_{2} / \mathrm{N}_{2}$ $(5: 95, \mathrm{v} / \mathrm{v})]$ which acted as an inoculum for an aerated second-stage [gas phase: $\mathrm{CO}_{2} /$ air $(5: 95, \mathrm{v} / \mathrm{v})$ ], in which the HA surfaces were inserted to allow biofilm development. This system was used to study the effect of oxygen and culture composition on the development of planktonic and biofilm communities. The study also investigated whether the 'obligately anaerobic' species could persist and/or grow in aerated conditions when part of a microbial community.

\section{METHODS}

Two-stage chemostat. A two-stage chemostat system was constructed using twin Anglicon (Brighton Systems) controllers, with a conventional culture vessel $(500 \mathrm{ml}$ working volume) connected via the overflow weir of the first-stage to a second-stage 'biofilm' vessel. 'The second-stage vessel $(750 \mathrm{ml}$ working volume) had a modified lid with multiple ports to allow the insertion and removal of indented $\mathrm{HA}$ discs $(7 \mathrm{~mm}$ diameter; Calcitek), which were suspended from rubber bungs with titanium wire, as described previously by Bradshaw et al. (1996).

Inoculum and growth conditions. Ten bacterial species were inoculated from a pooled inoculum, stored in the gas phase above liquid nitrogen, as described previously by Bradshaw et al. $(1989 \mathrm{~b})$, into the first-stage chemostat. The bacteria were Streptococcus mutans R9, Streptococcus oralis EF186, Streptococcus sanguis 209, Lactobacillus casei AC413, Actinomyces naeslundii WVU627, Neisseria subflava A1078, Veillonella dispar ATCC 17745, Porphyromonas gingivalis W50, Prevotella nigrescens T588 and Fusobacterium nucleatum ATCC 10953. The inoculum included facultative anaerobes $(N$. subflava, $S$. mutans, $S$. oralis, $S$. sanguis, $A$. naeslundii, $L$. casei) and bacteria which are conventionally regarded as obligate anaerobes ( $V$. dispar, Por. gingivalis, Pre. nigrescens, $F$. nucleatum). The chemostat growth medium was diluted BM medium (McKee et al., 1985) supplemented with hog gastric mucin (BMHGM; Bradshaw et al., 1989a), supplied to the culture at a rate of $50 \mathrm{ml} \mathrm{h}^{-1}$ (corresponding to a dilution rate, $D$, of $0 \cdot 1 \mathrm{~h}^{-1}$, and a mean generation time of $6 \cdot 9 \mathrm{~h}$ ). The chemostat was maintained automatically at $37 \pm 0 \cdot 1^{\circ} \mathrm{C}$ and $\mathrm{pH}$ was controlled at $7 \cdot 0 \pm 0.1$ by the automatic addition of $2 \mathrm{M}$ $\mathrm{NaOH}$, with a gas phase of $5 \% \mathrm{CO}_{2}$ in $\mathrm{N}_{2}$ (Bradshaw et al., 1989a). Once this culture had reached steady-state (after 4-5 d at $D=0 \cdot 1 \mathrm{~h}^{-1}$ ), the overflow weir of the first-stage was connected to the second-stage 'biofilm' chemostat, into which HA discs could be inserted for varying time periods. The gas phase in the second chemostat was $5 \% \mathrm{CO}_{2}$ in air, sparged at a rate of $200 \mathrm{ml}$ $\mathrm{min}^{-1}$. BMHGM medium was supplied to the second-stage vessel at $75 \mathrm{ml} \mathrm{h}^{-1}$ to give a total $D$ in the second-stage chemostat of $0.17 \mathrm{~h}^{-1}$, and the $\mathrm{pH}$ was again controlled at $7 \cdot 0 \pm 0 \cdot 1$. Dissolved oxygen tension $\left(\mathrm{dO}_{2}\right)$ was monitored with a polarographic electrode (Uniprobe). Following a period to allow the second-stage vessel to reach steady-state (at least $10 \times$ mean generation time, around 3-4d), HA discs were inserted, and planktonic sampling was commenced.

In a subsequent experiment, the primary oxygen-utilizing species in the culture, $N$. subflava, was omitted from the inoculum, so that a nine-membered culture developed. Finally, the system was reconfigured to reduce the rate of addition of first-stage culture to the second, aerated phase, to $5 \mathrm{ml} \mathrm{h}^{-1}$ (i.e. $1 / 10$ of the overflow from the first-stage chemostat), thus giving a total $D$ of $0 \cdot 11 \mathrm{~h}^{-1}$. Once again, a steady state (at least 3-4 d) was established prior to commencing experiments. The purpose of this experiment was to determine whether the anaerobic species were actually growing in the aerated phase.

Community composition. The bacterial composition of biofilm and planktonic communities was determined by decimal dilution of samples, followed by viable counts on a range of selective and non-selective agar media, as described previously by Bradshaw et al. (1993).

Statistical methods. Viable counts were calculated as c.f.u. $\mathrm{ml}^{-1}$. For the purposes of expressing data, a logarithmic transformation of data was performed, and means of $\log _{10}$ (c.f.u. $\left.\mathrm{ml}^{-1}\right) \pm$ standard deviation (SD) were calculated. Direct statistical comparisons between experiments were not possible because each experiment represented a single run of the chemostat. However, previous studies (Bradshaw et al., 1993, 1996) have indicated the reproducibility of this system, and therefore qualitative comparisons were made on this basis. Comparisons of the longitudinal trends in biofilm development within each run were carried out by the nonparametric Jonckheere-Terpstra test (Hollander \& Wolfe, 1973), using StatXact software (Cytel).

\section{RESULTS}

\section{Community development in a ten-membered, aerated bacterial culture}

The numbers of each species in planktonic cultures and biofilms in the aerated, second-stage chemostat vessel are shown in Table 1 as $\log _{10}$ (c.f.u $\mathrm{ml}^{-1}$ ) or $\log _{10}$ (c.f.u. $\left.\mathrm{cm}^{-2}\right) \pm \mathrm{SD}$, respectively. The planktonic culture was 
Table 1. Composition of the planktonic and biofilm communities in an aerated second-stage chemostat

Results are shown as $\log _{10}$ (c.f.u. $\mathrm{ml}^{-1}$ ) (culture) or $\log _{10}$ (c.f.u. $\mathrm{cm}^{-2}$ ) (biofilms) $\pm \mathrm{sD}$. The percentage of total c.f.u. values are shown in parentheses; $n=$ number of determinations within a chemostat run.

\begin{tabular}{|c|c|c|c|c|c|}
\hline \multirow[t]{2}{*}{ Species } & \multicolumn{5}{|c|}{ Bacterial numbers } \\
\hline & $\begin{array}{l}\text { Culture steady } \\
\text { state }(n=6)\end{array}$ & 1 h biofilm $(n=3)$ & $1 \mathrm{~d}$ biofilm $(n=3)$ & 4 d biofilm $(n=3)$ & 7 d biofilm $(n=3)$ \\
\hline S. mutans & $5.05 \pm 0.92(0.08 \%)$ & $4.94 \pm 0.27(0.5 \%)$ & $4.08 \pm 0.59(0.2 \%)$ & $4 \cdot 28 \pm 0 \cdot 13(0 \cdot 1 \%)$ & $4.76 \pm 0.41(0.2 \%)$ \\
\hline S. oralis & $6.56 \pm 0.26(2.7 \%)$ & $5.39 \pm 0.13(1.3 \%)$ & $5.62 \pm 0.27(7.0 \%)$ & $5 \cdot 42 \pm 0 \cdot 10(1.4 \%)$ & $5.99 \pm 0.32(2.6 \%)$ \\
\hline S. sanguis & $6.33 \pm 0.67(1.6 \%)$ & $5.32 \pm 0.09(1.1 \%)$ & $5 \cdot 10 \pm 0.05(2 \cdot 1 \%)$ & $5 \cdot 24 \pm 0 \cdot 15(0 \cdot 9 \%)$ & $5.69 \pm 0.08(1.3 \%)$ \\
\hline L. casei & $4.53 \pm 0.93(0.03 \%)$ & $4 \cdot 31 \pm 0 \cdot 30(0 \cdot 1 \%)$ & $3.77 \pm 0.98(0.1 \%)$ & $3.15 \pm 0.09(0.007 \%)$ & $4.66 \pm 0.35(0.1 \%)$ \\
\hline A. naeslundii & $4.20 \pm 0.41(0.01 \%)$ & $3.21 \pm 0.46(0.009 \%)$ & $3.99 \pm 0.48(0.2 \%)$ & $3.83 \pm 0.53(0.04 \%)$ & $3.96 \pm 1.07(0.02 \%)$ \\
\hline Por. gingivalis & $7 \cdot 17 \pm 0 \cdot 16(11 \cdot 1 \%)$ & $5.63 \pm 0.29(2.9 \%)$ & $5.90 \pm 0.19(13.4 \%)$ & $6.45 \pm 0.34(14.9 \%)$ & $6.99 \pm 0.13(25.6 \%)$ \\
\hline Pre. nigrescens & $7 \cdot 10 \pm 0 \cdot 26(9 \cdot 4 \%)$ & $5.77 \pm 0.28(3.2 \%)$ & $5.96 \pm 0.12(15.4 \%)$ & $6.04 \pm 0.47(5.8 \%)$ & $7.02 \pm 0.21(27.4 \%)$ \\
\hline F. nucleatum & $7 \cdot 44 \pm 0 \cdot 11(20 \cdot 6 \%)$ & $6.10 \pm 0.23(6.8 \%)$ & $5.93 \pm 0.21(14.3 \%)$ & $6.59 \pm 0.32(20.6 \%)$ & $6.81 \pm 0.31(16.9 \%)$ \\
\hline$V$. dispar & $7.04 \pm 0.20(8.2 \%)$ & $5.45 \pm 0.38(1.5 \%)$ & $5.61 \pm 0.20(6.9 \%)$ & $5 \cdot 82 \pm 0.10(3.5 \%)$ & $6.48 \pm 0.29(7.9 \%)$ \\
\hline N. subflava & $7.79 \pm 0.90(46.2 \%)$ & $7 \cdot 19 \pm 0.23(83.2 \%)$ & $6.38 \pm 0.20(40.4 \%)$ & $7 \cdot 00 \pm 0.22(52 \cdot 8 \%)$ & $6.84 \pm 0.35(18.1 \%)$ \\
\hline Total c.f.u. & $8 \cdot 125$ & $7 \cdot 270$ & 6.773 & $7 \cdot 277$ & $7 \cdot 616$ \\
\hline
\end{tabular}

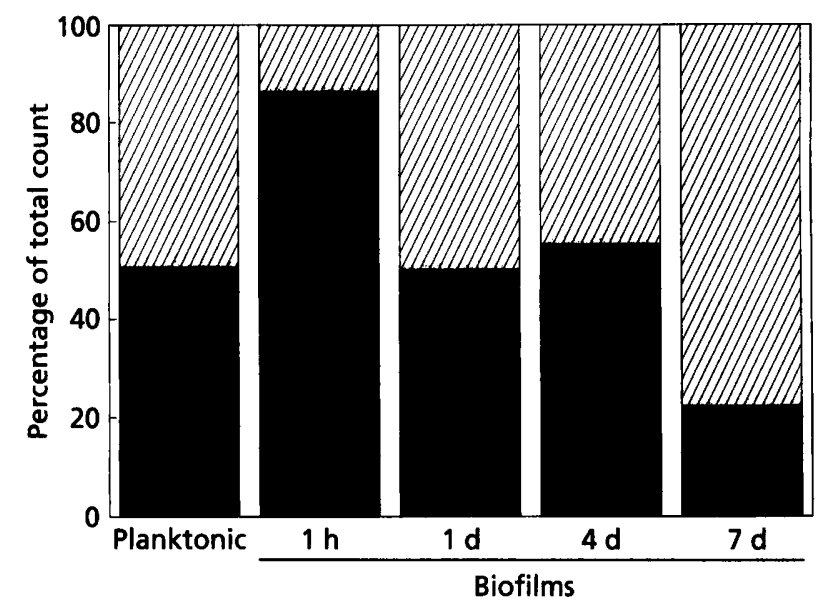

Fig. 1. Proportion of anaerobic and facultative species in planktonic culture and biofilms in a 10-species, aerated mixed culture. $\square$, Facultative anaerobes; $\square$, obligate anaerobes; $100 \%$ values correspond to total c.f.u. shown in Table 1.

dominated by $N$. subflava, which comprised more than $45 \%$ of the total c.f.u. at steady-state. Anaerobic species, especially Por. gingivalis and F. nucleatum remained in relatively high numbers in the culture, despite the constant sparging with $200 \mathrm{ml} 5 \% \mathrm{CO}_{2}$ in air min $^{-1}$, while the numbers of streptococci, $L$. case $i$ and $A$. naeslundii were low. The early biofilms $(1 \mathrm{~h})$ consisted mainly of $N$. subflava ( $>80 \%$ of total c.f.u.), although later biofilms, particularly after $7 \mathrm{~d}$, were predominated by anaerobes ( $>70 \%$ of c.f.u.), while the proportion of N. subflava was reduced. The proportions of anaerobes increased significantly with time in biofilms (Jonckheere-Terpstra test, $P<0.03$ ). The levels of streptococci in all biofilms remained low. Fig. 1 summarizes the overall shifts in proportions of strict anaerobes (Por. gingivalis, F. nucleatum, Pre. nigrescens, $V$. dispar) observed during biofilm development, compared with the steady-state planktonic phase.

Despite the relatively rapid rate of addition of oxygen to the chemostat culture, the $\mathrm{dO}_{2}$ at steady state remained low ( $<5 \%$ of air saturation). The steady-state $E_{\mathrm{h}}$ of the culture was -250 to $-300 \mathrm{mV}$.

\section{Community development in the absence of $N$. subflava}

As N. subflava had predominated within the early biofilms, presumably in response to the aeration of the environment, the effect of deleting this more aerotolerant species from the inoculum on community development was investigated. The composition of planktonic cultures and biofilms in the aerated chemostat without $N$. subflava is shown in Table 2. The planktonic culture was predominated by $S$. oralis, $F$. nucleatum, Pre. nigrescens, $S$. mutans and $V$. dispar. Although anaerobic bacteria remained in relatively high numbers in planktonic culture (Table 2), the count of Por. gingivalis was lower than that found in the culture which included N. subflava (Table 1).

Numbers of bacteria in young biofilms ( $2 \mathrm{~h}$ and $4 \mathrm{~h}$ ) without $N$. subflava were lower compared with $1 \mathrm{~h}$ biofilms developed in the culture with $N$. subflava (compare Tables 2 and 1 ), but had increased proportions of streptococci. These differences were not statistically tested. The proportions of anaerobes were again increased in later biofilms ( $1 \mathrm{~d}$ and $4 \mathrm{~d})$, though this increase only bordered on significance (Jonckheere-Terpstra test, $P=$ 
Table 2. Composition of the planktonic and biofilm communities in an aerated second-stage chemostat in which N. subflava had been omitted from the culture

Results are shown as $\log _{10}$ (c.f.u. $\mathrm{ml}^{-1}$ ) (culture) or $\log _{10}$ (c.f.u. $\mathrm{cm}^{-2}$ ) (biofilms) \pm sD. The percentage of total c.f.u. values are shown in parentheses; $n=$ number of determinations within a chemostat run.

\begin{tabular}{|c|c|c|c|c|c|}
\hline \multirow[t]{2}{*}{ Species } & \multicolumn{5}{|c|}{ Bacterial numbers } \\
\hline & $\begin{array}{c}\text { Culture steady } \\
\text { state }(n=5)\end{array}$ & 2 h biofilm $(n=3)$ & 4 h biofilm $(n=1)$ & $1 \mathrm{~d}$ biofilm $(n=1)$ & 4 d biofilm $(n=2)$ \\
\hline S. mutans & $7 \cdot 30 \pm 0 \cdot 10(15 \cdot 6 \%)$ & $5.64 \pm 0.04(15.2 \%)$ & $5 \cdot 36(7 \cdot 1 \%)$ & $5.85(13.8 \%)$ & $6 \cdot 11 \pm 0.30(4.8 \%)$ \\
\hline S. oralis & $7.51 \pm 0.08(25.3 \%)$ & $5.74 \pm 0.06(19.2 \%)$ & $5.77(18 \cdot 3 \%)$ & $5.94(17.0 \%)$ & $6 \cdot 30 \pm 0 \cdot 22(7 \cdot 4 \%)$ \\
\hline S. sanguis & $6.27 \pm 0.78(1.4 \%)$ & $4 \cdot 99 \pm 0 \cdot 21(3 \cdot 4 \%)$ & $4.45(0.9 \%)$ & $5 \cdot 15(2 \cdot 8 \%)$ & $4.99 \pm 0.43(0.4 \%)$ \\
\hline L. casei & $5.31 \pm 0.47(0.2 \%)$ & $4.31 \pm 0.65(0.7 \%)$ & $4 \cdot 75(1 \cdot 7 \%)$ & $4 \cdot 75(1 \cdot 1 \%)$ & $5.37 \pm 0.32(0.9 \%)$ \\
\hline A. naeslundii & $5 \cdot 64 \pm 1 \cdot 26(0.3 \%)$ & $4.43 \pm 0.58(0.9 \%)$ & $4.60(1 \cdot 2 \%)$ & $4.71(1.0 \%)$ & $3.81 \pm 1.16(0.02 \%)$ \\
\hline Por. gingivalis & $6.72 \pm 0.71(4.1 \%)$ & $4.48 \pm 0.43(1.1 \%)$ & $3.93(0.3 \%)$ & $4 \cdot 75(1 \cdot 1 \%)$ & $5 \cdot 84 \pm 0 \cdot 12(2 \cdot 6 \%)$ \\
\hline Pre. nigrescens & $7 \cdot 33 \pm 0.43(16.7 \%)$ & $5.94 \pm 0.15(30.4 \%)$ & $5.88(23.5 \%)$ & $6.14(26.9 \%)$ & $7.06 \pm 0.08(42.6 \%)$ \\
\hline F. nucleatum & $7 \cdot 45 \pm 0.09(22.0 \%)$ & $5 \cdot 70 \pm 0 \cdot 12(17 \cdot 5 \%)$ & $5.94(27 \cdot 0 \%)$ & $6.10(24.6 \%)$ & $6.86 \pm 0.11(26.9 \%)$ \\
\hline$V$. dispar & $7 \cdot 26 \pm 0.09(14 \cdot 2 \%)$ & $5 \cdot 52 \pm 0 \cdot 13(11 \cdot 6 \%)$ & $5 \cdot 81(20 \cdot 0 \%)$ & $5 \cdot 78(11 \cdot 8 \%)$ & $6.59 \pm 0.09(14.4 \%)$ \\
\hline Total & $8 \cdot 1066$ & 6.467 & $6 \cdot 502$ & 6.709 & $7 \cdot 431$ \\
\hline
\end{tabular}

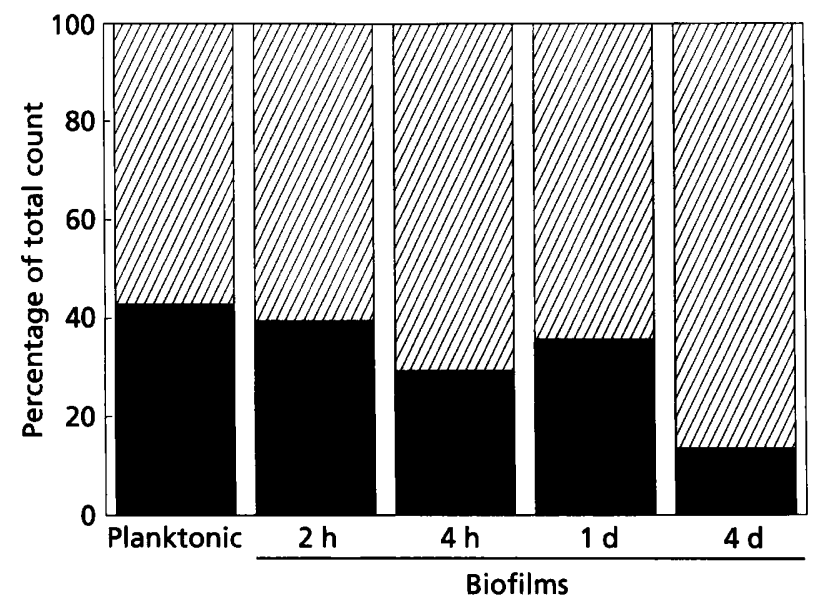

Fig. 2. Proportion of anaerobic and facultative species in planktonic culture and biofilms in a nine-species, aerated mixed culture, in which $N$. subflava was omitted. Facultative anaerobes; $\square$, obligate anaerobes; $100 \%$ values correspond to total c.f.u. shown in Table 2.

0.052). Fig. 2 summarizes the data in terms of proportions of strict anaerobes in developing biofilm populations and those persisting in the steady-state planktonic culture. The total count of the biofilm communities was lower in the absence of $N$. subflava during the first $4 \mathrm{~d}$ of development (Table 2 versus Table 1), though the difference observed was within the range found for similar biofilms in a previous study (Bradshaw et al., 1996).

In this culture, in contrast to that incorporating $N$. subflava, the $\mathrm{dO}_{2}$ was around $50-60 \%$ of air saturation, and the $E_{\mathrm{h}}$ was around $+50 \mathrm{mV}$.

\section{Community development in the absence of $N$. subflava, with reduced flow rate}

The data above established unequivocally that 'obligately anaerobic' species persisted in a microbial community grown in an aerated environment. The rate of addition of first-stage culture containing these anaerobes $\left(50 \mathrm{ml} \mathrm{h}^{-1}\right)$ in the experiment described above, however, made it impossible to establish categorically whether the anaerobes were actually growing in the aerated stage or were present simply as a consequence of the volume of firststage culture added. In order to clarify this, the system was reconfigured so that the rate of addition of first-stage culture to the second-stage was reduced to $1 / 10$ of that of the previous experiment. The composition of planktonic cultures and biofilms from this experiment is shown in Table 3. The planktonic culture was again predominated by the three streptococcal species, F. nucleatum, Pre. nigrescens and $A$. naeslundii. The total planktonic population was reduced by approximately threefold compared with the study in which $50 \mathrm{ml}$ conventional culture $\mathrm{h}^{-1}$ was added. This reduction appeared to be quantitative, with the proportions of different bacteria, including the anaerobic species, remaining relatively unaffected. The numbers of bacteria in young biofilms were reduced compared with the $50 \mathrm{ml} \mathrm{h}^{-1}$ addition culture $\left(10^{5 \cdot 8} \mathrm{~cm}^{-2}\right.$ versus $10^{6 \cdot 5} \mathrm{~cm}^{-2}$ for $2 \mathrm{~h}$ biofilms) (Tables 2 and 3 ) and this difference was greater than that seen in biofilms of this age previously (Bradshaw et al., 1996). These differences were less notable in older biofilms, and after $7 \mathrm{~d}$ the numbers were comparable with those seen in the culture including N. subflava (Tables 1 and 3 ). Bacterial succession occurred, with the proportions of anaerobes increasing significantly in older biofilms (Jonckheere-Terpstra test, $P<0.0003$ ), notably after 4 and $7 \mathrm{~d}$ growth. Fig. 3 summarizes these shifts, in comparison with the steady-state planktonic 
Table 3. Composition of the planktonic and biofilm communities in an aerated second-stage chemostat (lacking $N$. subflava), in which the rate of culture addition was reduced tenfold

Results are shown as $\log _{10}$ (c.f.u. $\mathrm{ml}^{-1}$ ) (culture) or $\log _{10}$ (c.f.u. $\mathrm{cm}^{-2}$ ) (biofilms) $\pm s D$. The percentage of total c.f.u. values are shown in parentheses; $n=$ number of determinations within a chemostat run.

\begin{tabular}{|c|c|c|c|c|c|c|}
\hline \multirow[t]{2}{*}{ Species } & \multicolumn{6}{|c|}{ Bacterial numbers } \\
\hline & $\begin{array}{l}\text { Culture steady state } \\
\qquad(n=6)\end{array}$ & 2 h biofilm $(n=3)$ & 4 h biofilm $(n=2)$ & $1 \mathrm{~d}$ biofilm $(n=3)$ & 4 d biofilm $(n=3)$ & 7 d biofilm $(n=3)$ \\
\hline S. mutans & $6.90 \pm 0.28(13.8 \%)$ & $5.04 \pm 0.38(18.8 \%)$ & $5 \cdot 25 \pm 0.28(9 \cdot 2 \%)$ & $5.55 \pm 0.04(11.6 \%)$ & $5.20 \pm 0.12(6.7 \%)$ & $6.46 \pm 0.31(12.5 \%)$ \\
\hline S. oralis & $6.99 \pm 0.16(17 \cdot 1 \%)$ & $5.03 \pm 0.40(18.3 \%)$ & $5.44 \pm 0.06(14.3 \%)$ & $5.84 \pm 0.15(22.7 \%)$ & $5.56 \pm 0.09(15.3 \%)$ & $6.36 \pm 0.31(9.9 \%)$ \\
\hline S. sanguis & $6.89 \pm 0.21(13.5 \%)$ & $5.08 \pm 0.30(20.6 \%)$ & $5.74 \pm 0.09(28.6 \%)$ & $5.62 \pm 0.18(13.7 \%)$ & $5.50 \pm 0.23(13.3 \%)$ & $5.93 \pm 0.13(3.7 \%)$ \\
\hline L. casei & $6.06 \pm 0.31(2.0 \%)$ & $4.10 \pm 0.29(2.2 \%)$ & $5.29 \pm 0.02(10 \cdot 1 \%)$ & $5.63 \pm 0.18(14.0 \%)$ & $5 \cdot 38 \pm 0.24(10 \cdot 1 \%)$ & $5.36 \pm 0.40(1.0 \%)$ \\
\hline A. naeslundii & $6.76 \pm 0.35(10.0 \%)$ & $5.02 \pm 0.37(17.9 \%)$ & $5.68 \pm 0.04(24.9 \%)$ & $5.57 \pm 0.36(12.2 \%)$ & $5.19 \pm 0.33(6.5 \%)$ & $5 \cdot 40 \pm 0 \cdot 27(1 \cdot 1 \%)$ \\
\hline Por. gingivalis & $6.69 \pm 0.42(8.5 \%)$ & $3.79 \pm 0.74(1.1 \%)$ & $3.93 \pm 0.25(0.4 \%)$ & $4.07 \pm 0.54(0.4 \%)$ & $4.28 \pm 0.76(0.8 \%)$ & $6.30 \pm 0.53(8.6 \%)$ \\
\hline Pre. nigrescens & $6.87 \pm 0.23(12.9 \%)$ & $4 \cdot 10 \pm 0.60(2 \cdot 2 \%)$ & $4.26 \pm 0.05(0.9 \%)$ & $4.77 \pm 0.56(1.9 \%)$ & $5.49 \pm 0.23(13.0 \%)$ & $6.88 \pm 0.36(32.9 \%)$ \\
\hline F. nucleatum & $6.96 \pm 0.96(15.9 \%)$ & $4.91 \pm 0.10(13.9 \%)$ & $5.14 \pm 0.02(7.2 \%)$ & $5.66 \pm 0.03(15.0 \%)$ & $5.75 \pm 0.20(23.7 \%)$ & $6.75 \pm 0.34(24.4 \%)$ \\
\hline$V$. dispar & $6.55 \pm 0.20(6.2 \%)$ & $4 \cdot 48 \pm 0 \cdot 30(5 \cdot 2 \%)$ & $4.92 \pm 0.07(4.3 \%)$ & $5 \cdot 41 \pm 0 \cdot 20(8 \cdot 4 \%)$ & $5 \cdot 40 \pm 0 \cdot 12(10 \cdot 6 \%)$ & $6.14 \pm 0.13(6.0 \%)$ \\
\hline Total & $7 \cdot 760$ & $5 \cdot 766$ & $6 \cdot 29$ & 6.484 & $6 \cdot 376$ & $7 \cdot 363$ \\
\hline
\end{tabular}

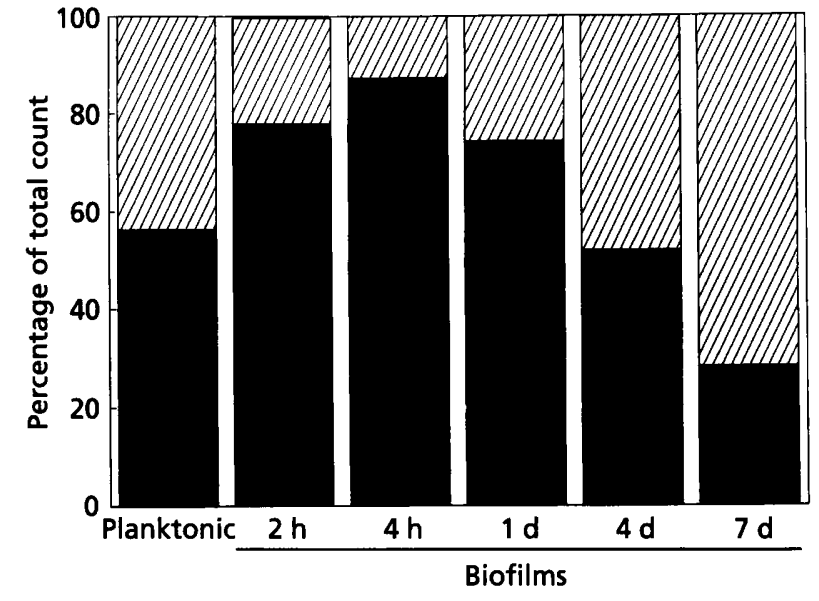

Fig. 3. Proportion of anaerobic and facultative species in planktonic culture and biofilms in a nine-species, aerated mixed culture, in which $N$. subflava had been omitted. Culture addition from the first-stage was at a reduced (1/10) flow rate. 口, Facultative anaerobes; $\square$, obligate anaerobes; $100 \%$ values correspond to total c.f.u. shown in Table 3.

culture. The oxygen tension in steady-state culture was between 25 and $50 \%$, and the measured $E_{\mathrm{h}}$ was between $+90 \mathrm{mV}$ and $+140 \mathrm{mV}$.

\section{DISCUSSION}

Obligately anaerobic species were able to persist in high numbers in a ten-membered mixed culture, both in the biofilm and in the planktonic phase, even when the culture was sparged with $5 \% \mathrm{CO}_{2}$ in air. Under these conditions, $N$. subflava was the major organism during the early stages of biofilm formation, and comprised $>45 \%$ of c.f.u. in the steady-state planktonic phase. The proportions of $N$. subflava found in young biofilms in this study were far higher compared with biofilms developed anaerobically, which were described in a previous study (Bradshaw et al., 1996). The anaerobic populations in biofilms in the present study increased significantly in proportion over time, but predominated only after 4-7 d. This is similar to the successional changes observed during the development of plaque in humans (Ritz, 1967; Hardie \& Bowden, 1976). The environmental conditions within the mixed culture remained relatively anaerobic, with the $\mathrm{dO}_{2}$ of the planktonic phase only just measurable, and with a highly negative $E_{h}$, despite continuous sparging with $5 \% \mathrm{CO}_{2}$ in air. This $E_{h}$ was as low as that which was measured in conventional anaerobic mixed cultures of oral bacteria (McKee et al., 1985). Since the numbers of $N$. subflava were so high, it was assumed that the growth and metabolism of this organism must be accounting for a substantial proportion of the oxygen consumption in the culture. The advantage of using a defined inoculum approach (Bradshaw et al., 1989b) was exploited to determine the significance of the role of $N$. subflava in providing an environment conducive to the growth of the strict anaerobes, by constructing a new, nine-species inoculum from which $N$. subflava was omitted. In the absence of this aerobe, obligate anaerobes were still able to persist in planktonic and biofilm communities, but were found in slightly lower numbers in the younger biofilms. The loss of $N$. subflava was compensated for by an increase in the total numbers of streptococci in both planktonic and biofilm phases, though this increase was not statistically tested. Streptococci were found in lower proportions in early biofilms than in de novo dental plaque, presumably reflecting their proportions in the planktonic phase in this model system. Anaerobic species increased significantly in proportion, so that after $4 \mathrm{~d}$ they were predominant. The assumption that $N$. subflava had been 
responsible for the majority of the oxygen consumption was supported by the observation that in the culture without N. subflava, oxygen was now readily detectable, and the $E_{\mathrm{h}}$ was positive. The persistence of bacteria conventionally regarded as obligate anaerobes in high numbers in such an aerated environment in both the planktonic and biofilm phases of growth was unexpected.

The experimental design had a conventional anaerobic chemostat feeding into an aerated second-stage that contained surfaces available for colonization. The rate of addition from the first-stage chemostat in the experiments discussed above made it difficult to determine to what extent the anaerobes were actually reproducing in the second-stage. When the rate of addition was reduced tenfold, the total numbers of anaerobes in the planktonic phase fell by less than threefold, confirming that they were not merely persisting in the culture, but were actually reproducing. These anaerobes were able to colonize the HA surfaces and eventually grow to predominate within the biofilm community after $7 \mathrm{~d}$. This indicated that in this mixed culture, anaerobes could reproduce in aerated conditions, presumably as a result of the presence of facultative species, as part of a microbial community. This is a comparable situation to the persistence of sulphatereducing bacteria in anaerobic micro-niches in biofilms associated with corrosion that develop on mild steel immersed in an aerated aqueous phase (Hamilton, 1987; Dexter, 1993; Lee et al., 1995). This type of interaction may also be one of the explanations for the almost ubiquitous occurrence of facultative and obligately anaerobic species in microbial consortia isolated from abscesses (Fabricus et al., 1982; Brook, 1985). Collectively, these findings address the issue that has been raised recently as to whether biofilms are true microbial communities or whether they represent more-or-less 'random accretions of organisms deposited in a haphazard manner on a solid surface, united only by their ability to adhere and proliferate in such an environment' (Wimpenny, 1994). Data from a range of habitats in the mouth imply that these oral biofilms are not arranged randomly; rather, they represent an organized and interactive consortium of micro-organisms. Such an arrangement enables individual species to combine metabolically to deal most efficiently with the prevailing physico-chemical conditions. This could be the utilization of oxygen (or the ability to persist in high redox environments), or the ability to act in a concerted manner to catabolize endogenous substrates (e.g. host glycoproteins) as nutrient sources (Bradshaw $e t$ al., 1994).

It is well established that oxygen gradients can exist within biofilms, with anoxic conditions being established close to the biofilm surface (Hamilton, 1987; Dexter, 1993). Depths of $8-12 \mu \mathrm{m}$ were sufficient for oxygen to become limiting in artificial biofilms of nitrifying bacteria (Cox et al., 1980) or oral bacteria (Coulter \& Russell, 1976). The biofilms in this system have a heterogeneous structure, with depths ranging from 5 to $22 \mu \mathrm{m}$ (Bradshaw et al., 1996). It may be that the older biofilms are capable of being organized spatially to protect the strict anaerobes within the community, thus resulting in their increasing predominance with time in all three experiments described in this study. Although no structural or species distribution studies were carried out on the present biofilms, N. subflava appeared to be predominant in the upper levels and F. nucleatum in the lower layers of biofilms generated from a similar inoculum in a constant-depth $(300 \mu \mathrm{m})$ film fermenter (Kinniment et al., 1996). Similarly, Neisseria spp. have been observed in the upper layers of mature human plaque whereas $V$ eillonella spp. were located in the deeper layers of the biofilm (Ritz, 1969). The observation that bacteria which, by normal criteria, are defined as obligate anaerobes can grow in aerated conditions in the planktonic phase is somewhat more difficult to explain. Kolenbrander (1988) demonstrated a number of highly specific coaggregations between oral bacteria, and has suggested that these could provide a mechanism by which successional development might occur during dental plaque development. This mechanism could also operate in the planktonic phase, and clumps of bacteria thus formed could then potentially protect anaerobic bacteria. The kinetics of oxygen consumption would be of key importance in ensuring gradient development over such short distances. Future studies will examine the mechanisms by which the obligately anaerobic bacteria might survive the conditions of aeration.

\section{ACKNOWLEDGEMENTS}

The authors would like to thank Eric Huntington (Unilever Research) for his assistance in the statistical interpretation of the data.

\section{REFERENCES}

Bradshaw, D. J. \& Marsh, P. D. (1994). Effect of sugar alcohols on the composition and metabolism of a mixed culture of oral bacteria grown in a chemostat. Caries Res 28, 251-256.

Bradshaw, D. J., McKee, A. S. \& Marsh, P. D. (1989a). Effect of carbohydrate pulses and $\mathrm{pH}$ on population shifts within oral microbial communities in vitro. $J$ Dent Res 68, 1298-1302.

Bradshaw, D. J., McKee, A. S. \& Marsh, P. D. (1989b). The use of defined inocula stored in liquid nitrogen for mixed-culture chemostat studies. J Microbiol Methods 9, 123-128.

Bradshaw, D. J., McKee, A. S. \& Marsh, P. D. (1990). Prevention of population shifts in oral microbial communities in vitro by low fluoride concentrations. J Dent Res 69, 436-441.

Bradshaw, D. J., Marsh, P. D., Watson, G. K. \& Cummins, D. (1993). The effects of Triclosan and zinc citrate, alone and in combination, on a community of oral bacteria grown in vitro. J Dent Res 72, 25-30.

Bradshaw, D. J., Homer, K. A., Marsh, P. D. \& Beighton, D. (1994). Metabolic cooperation in oral microbial communities during growth on mucin. Microbiology 140, 3407-3412.

Bradshaw, D. J., Marsh, P. D., Schilling, K. M. \& Cummins, D. (1996). A modified chemostat system to study the ecology of oral biofilms. J Appl Bacteriol 78 (in press).

Brook, I. (1985). Enhancement of growth of aerobic and facultative bacteria in mixed infections with Bacteroides species. Infect Immun 50, 929-931.

Costerton, J. W., Cheng, K. J., Geesey, G. G., Ladd, T. I., Nickel, J. C., Dasgupta, M. \& Marrie, T. J. (1987). Bacterial biofilms in nature and disease. Annu Rev Microbiol 41, 435-464. 
Coulter, W. A. \& Russell, C. (1976). $\mathrm{pH}$ and $\mathrm{E}_{\mathrm{h}}$ in single and mixed culture bacterial plaque in an artificial mouth. J Appl Bacteriol 40, 73-81.

Cox, D. J., Bazin, M. J. \& Gull, K. (1980). Distribution of bacteria in a continuous-flow nitrification system. Soil Biol Biochem 12, 241-246.

Dexter, S. C. (1993). Rôle of microfouling organisms in marine corrosion. Biofouling 7, 97-127.

Fabricus, L., Dahlen, G., Holm, S. E. \& Möller, J. R. (1982). Influence of combinations of oral bacteria on periapical tissues of monkeys. Scand J Dent Res 90, 200-206.

Hamilton, W. A. (1987). Biofilms: microbial interactions and metabolic activities. In Ecology of Microbial Communities, pp. 13-17. Edited by M. Fletcher, T. R. J. Gray \& J. G. Jones. Cambridge: Cambridge University Press.

Hardie, J. M. \& Bowden, G. H. (1976). The microbial flora of dental plaque: bacterial succession and isolation considerations. In Proceedings: Microbial Aspects of Dental Caries. Special Supplements to Microbiology Abstracts, pp. 63-98. Edited by H. M. Stiles, W. J. Loesche \& T. C. O'Brien. Washington, DC: Information Retrieval.

Hollander, M. \& Wolfe, D. A. (1973). Nonparametric statistical methods. New York: John Wiley.

Kinniment, S. L., Wimpenny, J. W. T., Adams, D. \& Marsh, P. (1996). Development of a steady-state oral microbial biofilm community using the constant-depth film fermenter. Microbiology 142, 631-638.

Kolenbrander, P. E. (1988). Intergeneric coaggregation among human oral bacteria and ecology of dental plaque. Annu Rev Microbiol 42, 627-656.

Lee, W., Lewandowski, Z., Nielsen, P. H. \& Hamilton, W. A. (1995). Role of sulfate-reducing bacteria in corrosion of mild steel: a review. Biofouling 8, 165-194.
McDermid, A. S., McKee, A. S., Ellwood, D. C. \& Marsh, P. D. (1986). The effect of lowering the $\mathrm{pH}$ on the composition and metabolism of a community of nine oral bacteria grown in a chemostat. J Gen Microbiol 132, 1205-1214.

McDermid, A. S., McKee, A. S. \& Marsh, P. D. (1987). A mixed culture chemostat system to predict the effect of antimicrobial agents on the oral flora: preliminary studies using chlorhexidine. J Dent Res 66, 1315-1320.

McKee, A. S., McDermid, A. S., Ellwood, D. C. \& Marsh, P. D. (1985). The establishment of reproducible, complex communities of oral bacteria in the chemostat using defined inocula. $J$ Appl Bacteriol 59, 263-275.

Marsh, P. D., Hunter, J. R., Bowden, G. H., Hamilton, I. R., McKee, A. S., Hardie, J. M. \& Ellwood, D. C. (1983). The influence of growth rate and nutrient limitation on the microbial composition and biochemical properties of a mixed culture of oral bacteria grown in a chemostat. J Gen Microbiol 129, 755-770.

Ritz, H. L. (1967). Microbial populations in developing human dental plaque. Arch Oral Biol 12, 1561-1568.

Ritz, H. L. (1969). Fluorescent staining of Neisseria, Streptococcus and Veillonella in frozen sections of human dental plaque. Arch Oral Biol 14, 1073-1083.

Wimpenny, J. W. T. (1994). The spatial organisation of biofilm. In Bacterial Biofilms and their Control in Medicine and Industry, pp. 1-6. Edited by J. Wimpenny, W. Nichols, D. Stickler \& H. LappinScott. Cardiff: Bioline.

Received 5 July 1995; revised 12 October 1995; accepted 24 October 1995. 